ELECTRONIC RESEARCH ANNOUNCEMENTS OF THE AMERICAN MATHEMATICAL SOCIETY

Volume 4, Pages 74-87 (October 22, 1998)

S $1079-6762(98) 00050-X$

\title{
DRINFEL'D DOUBLES AND EHRESMANN DOUBLES FOR LIE ALGEBROIDS AND LIE BIALGEBROIDS
}

\author{
K. C. H. MACKENZIE
}

(Communicated by Frances Kirwan)

\begin{abstract}
We show that the Manin triple characterization of Lie bialgebras in terms of the Drinfel'd double may be extended to arbitrary Poisson manifolds and indeed Lie bialgebroids by using double cotangent bundles, rather than the direct sum structures (Courant algebroids) utilized for similar purposes by Liu, Weinstein and $\mathrm{Xu}$. This is achieved in terms of an abstract notion of double Lie algebroid (where "double" is now used in the Ehresmann sense) which unifies many iterated constructions in differential geometry.
\end{abstract}

\section{INTRODUCTION}

It is well known that Lie bialgebras may be characterized in terms of Manin triples: a Lie algebra $\mathfrak{g}$ with a Lie algebra structure on the dual $\mathfrak{g}^{*}$ is a Lie bialgebra if and only if the vector space direct sum $\mathfrak{g} \oplus \mathfrak{g}^{*}$ has a Lie algebra structure making $\mathfrak{g}$ and $\mathfrak{g}^{*}$ isotropic subalgebras and making the natural pairing of $\mathfrak{g} \oplus \mathfrak{g}^{*}$ with itself invariant [7]. For a general Poisson manifold $P$, both $T P$ and $T^{*} P$ have Lie algebroid structures and it is reasonable to seek a version of the Manin triple result for this case and, more generally, for Lie bialgebroids in the sense of the author and $\mathrm{Xu}[23]$.

One can quickly see that the direct transposition of the notion of Manin triple to the Lie bialgebroid $\left(T P, T^{*} P\right)$ is only valid under very restrictive conditions on $P$. Very recently Liu, Weinstein and $\mathrm{Xu}[14]$ have given a highly nontrivial development of the notion of Manin triple to the bialgebroid case. For a Lie bialgebroid $\left(A, A^{*}\right)$ they define a bracket structure on $\Gamma\left(A \oplus A^{*}\right)$ which is recognizably an extension of the Manin formula and which, though it is not itself usually a Lie algebroid bracket, induces the given brackets on $\Gamma A$ and $\Gamma A^{*}$. The properties of this bracket on $E=A \oplus A^{*}$ are abstracted by [14] into a notion called Courant algebroid, and those Courant algebroids $E$ which arise from Lie bialgebroids are characterized as those which possess a transversal pair of integrable isotropic subbundles $L_{1}, L_{2} \subseteq E$; these are then $A, A^{*}$ for a Lie bialgebroid structure.

We argue here that it is also valid, and in certain senses more natural, to regard the double of a Poisson manifold $P$ (by which we mean the double of its Lie bialgebroid) as $T^{*} T P \cong T^{*} T^{*} P$ rather than $T P \oplus T^{*} P$. More generally, we provide a detailed justification for regarding the double of a Lie bialgebroid $\left(A, A^{*}\right)$

Received by the editors July 12, 1998.

1991 Mathematics Subject Classification. Primary 58F05; Secondary 17B66, 18D05, 22A22, $58 \mathrm{H} 05$.

(C)1998 American Mathematical Society 
as not $A \oplus A^{*}$ but $T^{*} A \cong T^{*} A^{*}$. Here $T^{*} A$ should be regarded as in $[23, \S 5]$ as the double vector bundle in Figure 1 , where $T^{*} A \rightarrow A$ is the usual cotangent

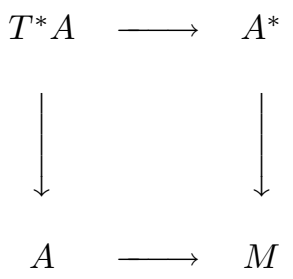

Figure 1.

bundle and $T^{*} A \rightarrow A^{*}$ is the composition of $T^{*} A^{*} \rightarrow A^{*}$ with the canonical antisymplectomorphism $R^{-1}: T^{*} A \rightarrow T^{*} A^{*}$. If $A$ is written locally as $M \times V$, then $R: T^{*} M \times V^{*} \times V \rightarrow T^{*} M \times V \times V^{*}$ interchanges $V$ with $V^{*}$, and is -id on $T^{*} M$; see $[23,5.5]$. We call Figure 1 the double cotangent bundle of $A$. If $A$ is a Lie algebroid, then $A^{*}$ has its dual Poisson structure and this induces a Lie algebroid structure on $T^{*} A^{*} \rightarrow A^{*}$. Likewise a Lie algebroid structure on $A^{*}$ induces a Lie algebroid structure on $T^{*} A \rightarrow A$. Thus Lie algebroid structures on $A$ and $A^{*}$ equip the four sides of Figure 1 with Lie algebroid structures. What we are seeking is an abstract set of compatibility conditions for these structures which, when applied to Figure 1, will characterize Lie bialgebroid stuctures on $A$. This is our first problem.

The key to this is the notion of double Lie groupoid in the sense of a Lie groupoid object in the category of Lie groupoids. This sense of the word "double" goes back to Ehresmann [9, III-1]; double and multiple structures in this sense have been used for many years in homotopy theory [3]. This use of the word should be carefully distinguished from the Drinfel'd sense [8], which we have been discussing until now, in which a double is a self-dual object composed from a fundamental structure and its dual. Nonetheless it is one consequence of this work that the two notions of double correspond in a sense which we will make precise.

In fact the presence of double groupoids underlying Poisson groups is already well established. Lu and Weinstein [16] proved that a Poisson group may always be integrated to a symplectic double groupoid and, slightly earlier, the same authors [17] and Majid [26] gave sufficent conditions for the dressing transformations of a Poisson Lie group to integrate globally; it was then shown in $[19, \S \S 2,4]$ that the twisted multiplicativity conditions of these global actions reflect exactly the fact that they come from a double groupoid structure. See $\S 2$ below.

The author's interest in a prospective Lie theory for double groupoids goes back to 1988, before he was properly aware of the relationships between Poisson geometry and groupoid and algebroid theory. As stated in the Introduction to [19], the principal purpose was to understand higher-order constructions in connection theory and more generally in differential geometry, specifically for rendering natural the apparent lack of structure on jet prolongation spaces by embedding them in iterated multiple structures of the type encompassed by the theory given here. This aspect is still of interest and will be treated elsewhere.

In order to proceed, we need to describe the notion of double Lie groupoid in more detail (see [19] and references given there). A double Lie groupoid consists of 
a manifold $S$ equipped with two Lie groupoid structures on bases $H$ and $V$, each of which is a Lie groupoid over a common base $M$, such that the structure maps (source, target, multiplication, identity, inversion) of each groupoid structure on $S$ are morphisms with respect to the other; see Figure 3. One should think of elements of $S$ as squares, the horizontal edges of which come from $H$, the vertical edges from $V$, and the corner points from $M$. Since $S$ is a Lie groupoid over $H$ we may take its Lie algebroid, called $A_{V} S$, and because the Lie functor preserves diagrams and pullbacks, $A_{V} S$ is a Lie groupoid over $A V$; see Figure 5(a). These two structures on $A_{V} S$ commute in the same way that the original groupoid structures on $S$ commute: each of the groupoid structure maps is a morphism of Lie algebroids. We call structures of this type $\mathcal{L} \mathcal{A}$-groupoids. In the case of a double groupoid arising from a Poisson group $G$ with dual group $G^{*}$, the $\mathcal{L} \mathcal{A}$-groupoids are $T^{*} G$ and $T^{*} G^{*}$. See $[19, \S 4]$.

Now one may take the Lie algebroid of the horizontal groupoid structure on $A_{V} S$ and obtain a double vector bundle $A^{2} S=A\left(A_{V} S\right)$ whose horizontal structure is a Lie algebroid. Interchanging the order of the processes - so that one first takes the Lie algebroid of the horizontal structure on $S$ and then the Lie algebroid of the resulting vertical structure - one obtains a second double vector bundle $A_{2} S=$ $A\left(A_{H} S\right)$ with the Lie algebroid structure now placed vertically. These two double vector bundles may be identified by a map derived from the canonical involution in the double tangent bundle of $S$, and one thus obtains a double vector bundle all four sides of which have Lie algebroid structures; this is what we call the double Lie algebroid of $S$. It includes, amongst many examples, the double tangent bundle of an arbitrary manifold, the Lie bialgebra of a Poisson group, and the double cotangent bundle of a Poisson manifold. See [20].

The construction of $A^{2} S \cong A_{2} S$ from $S$ ensures that the four Lie algebroid structures on the sides of Figure $5(\mathrm{~b})$ are suitably compatible. Our second problem is to isolate these compatibility conditions and give a general definition of a double Lie algebroid without reference to any underlying double groupoid.

We will see that the solutions to these two problems are closely related.

The difficulty is that whereas the notions of double groupoid and of $\mathcal{L} \mathcal{A}$-groupoid can be defined by morphism conditions on the structure maps of a groupoid, this is not possible with double Lie algebroids. The bracket of a Lie algebroid is defined not on the bundle itself but on its module of sections and it is not clear what could be meant by requiring one bracket to be a morphism with respect to the other. Instead we proceed by an indirect route, which makes essential use of the duality between Lie algebroids and (fibrewise linear) Poisson structures. Thus summarized, this strategy may sound straightforward, but its implementation is not: the duals to be taken are duals of double and triple structures and have many unexpected features.

This is a summary and overview of [20], [21], and [22], together with [19]. I am grateful to many people for comments on various stages of the work, acknowledged in the individual papers, and to Yvette Kosmann-Schwarzbach also for comments on this announcement. Overall I especially want to thank Ronnie Brown, Alan Weinstein, and Ping $\mathrm{Xu}$. 


\section{Double Lie Groupoids AND Associated LiE AlgEBroids}

It is valuable to keep in mind the very classical examples of the double (or iterated) tangent bundle $T^{2} M$ of a manifold $M$, as in Figure 2(a), and more generally the tangent bundle $T A$ of an arbitrary vector bundle $(A, q, M)$, as in Figure $2(\mathrm{~b})$.

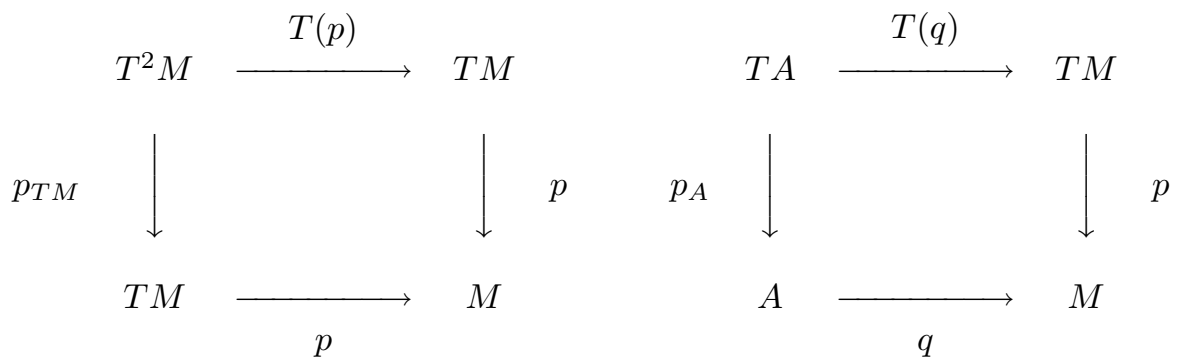

(a)

FiguRE 2 .

(b)

As well as its standard tangent bundle structure over $A$, there is a prolonged vector bundle structure $(T A, T(q), T M)$ obtained by applying the tangent functor to the bundle projection, the zero section, the addition, and the scalar multiplication, of $A$. This is a double vector bundle in the sense of [28], [19, §1]; that is, the structure maps of each structure on $T A$ are morphisms with respect to the other structure. For a traditional treatment, see [2].

Recalling the definition sketched in the Introduction, we picture a double Lie groupoid $(S ; H, V ; M)$ and a typical element as in Figure 3 ; here $\alpha$ and $\beta$ generically

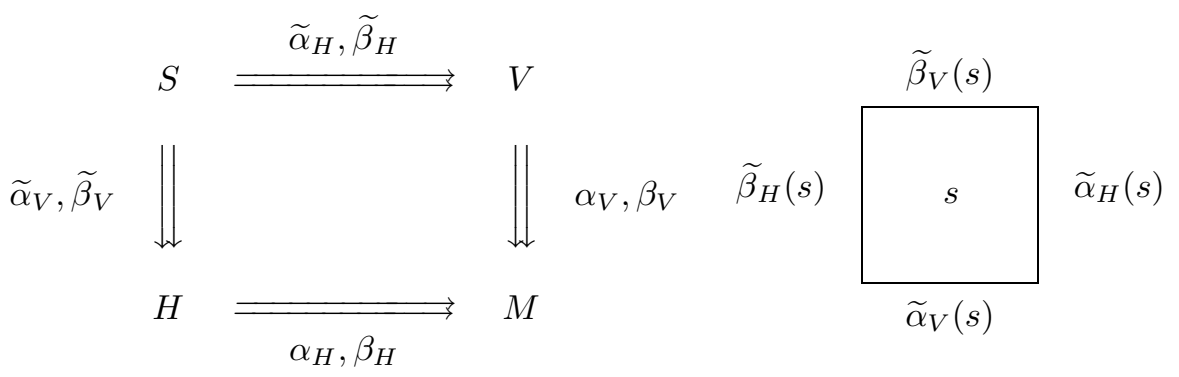

FIGURE 3.

denote source and target projections respectively.

The definition ensures that when a composite of four elements as in Figure 4 may be calculated as $\left(s_{2} \square s_{1}\right) \boxminus\left(s_{3} \square s_{4}\right)$, that is, first composing horizontally and then vertically, then it may also be calculated as $\left(s_{2} \boxminus s_{3}\right) \square\left(s_{1} \boxminus s_{4}\right)$, and the results are equal. The smoothness condition ensures (see $[4,1.2]$ ) that both structures on $S$ are Lie groupoids (called differentiable groupoids in [18]) and that the Lie functor may be applied to the structure maps. We will describe examples below.

Applying the Lie functor to the vertical structure on $S$ we obtain Figure 5(a). Since the Lie functor preserves pullbacks, $A_{V} S$, the Lie algebroid of $S \Longrightarrow H$, has a prolonged Lie groupoid structure over $A V$. See $[19, \S 4]$. Now $A^{2} S=A\left(A_{V} S\right)$ in (b) is the Lie algebroid of this Lie groupoid. Using the Lie functor in the same way as the tangent functor is used in Figure 2, $A^{2} S$ has a vector bundle structure over 


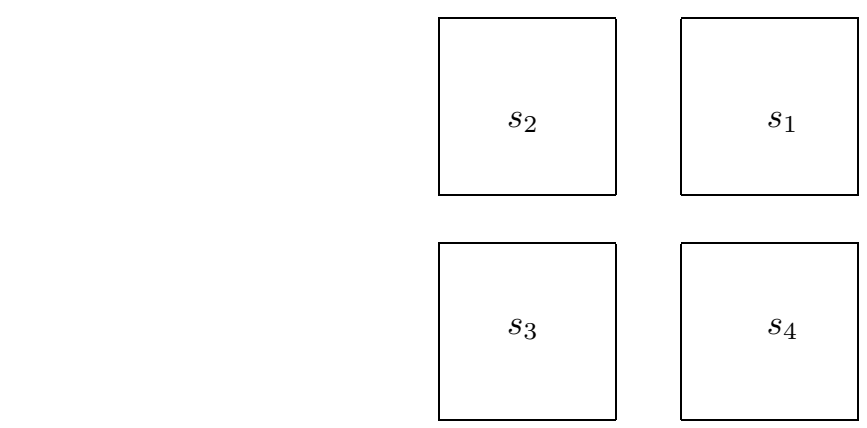

FiguRE 4.

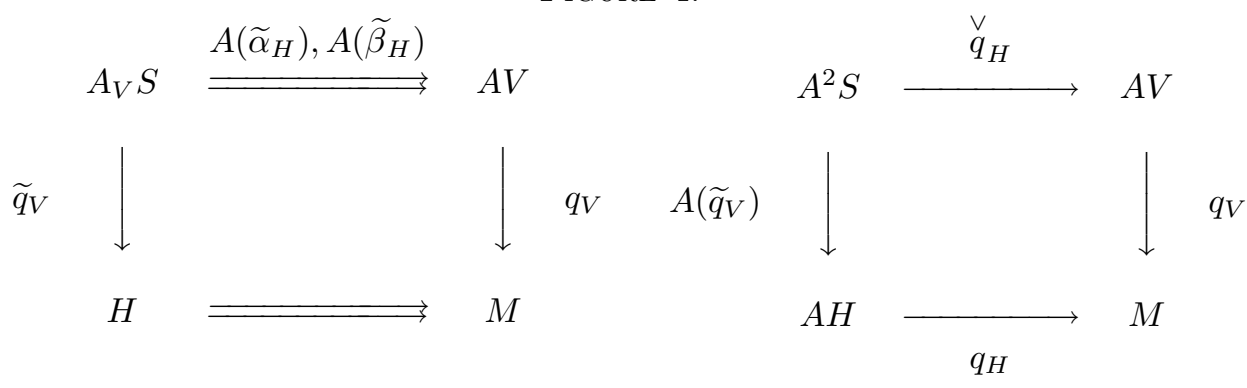

(a)

Figure 5.

(b)

$A H$. Thus $A^{2} S$ is a double vector bundle with Lie algebroid structures on three of its four sides. See $[20, \S 2]$.

We can likewise take first the horizontal Lie algebroid, obtaining a Lie groupoid $A_{H} S \Longrightarrow A H$ as in Figure 6(a), and then define $A_{2} S=A\left(A_{H} S\right)$ as in Figure 6(b).

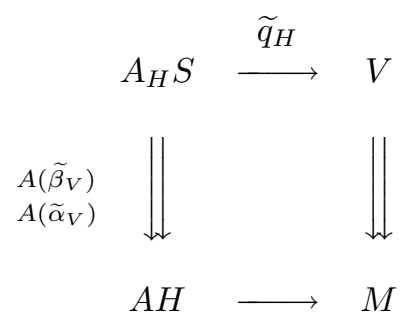

(a)

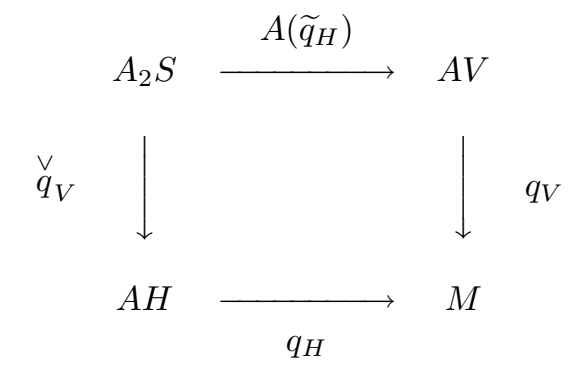

(b)

Now the canonical involution $J_{S}: T^{2} S \rightarrow T^{2} S$ restricts to give an isomorphism of double vector bundles $j_{S}: A^{2} S \rightarrow A_{2} S$. Using this we transport the Lie algebroid structure of $A_{2} S \rightarrow A H$ back to give $A^{2} S$ four Lie algebroid structures. Thus equipped, $A^{2} S$ is the double Lie algebroid of $(S ; H, V ; M)$. See $[20, \S 2]$.

Example 2.1. Taking $H=V=M \times M$ to be the pair groupoid, and $S=M^{4}$ to be the double groupoid whose elements are the four corners of empty squares, we have that $A_{V} S$ is the pair groupoid $T M \times T M$, and so $A^{2} S=T^{2} M$, with horizontal structure the tangent of $T M$ and vertical structure the tangent prolongation of 
$T M \rightarrow M$. Likewise $A_{2} S$ is $T^{2} M$ with the horizontal and vertical structures interchanged.

More generally, take $S=G \times G$ where $G \Longrightarrow M$ is any Lie groupoid and $S$ is horizontally the pair groupoid over $G$ and vertically the Cartesian square groupoid over $M \times M$. Then $A_{H} S=T G$ is the tangent groupoid over $T M$ and $A_{2} S=A T G$, the Lie algebroid of the tangent groupoid. On the other hand, $A_{V} S=A G \times A G$, the pair groupoid, and $A^{2} S=T A G$ the tangent prolongation, defined intrinsically in $[23, \S 5]$, of the Lie algebroid $A G \rightarrow M$. The canonical isomorphism $j_{G}: T A G \rightarrow$ $A T G$ is described in $[23, \S 7]$.

An important class of examples presents naturally as structures intermediate between double Lie groupoids and double Lie algebroids. An $\mathcal{L} \mathcal{A}$-groupoid $[19$, $\S 4](\Omega ; G, A ; M)$, as in Figure $7(\mathrm{a})$, comprises both a Lie groupoid structure on $\Omega$ over base $A$ (which is itself a Lie algebroid over $M$ ), and a Lie algebroid on $\Omega$ over $G$ (which is a Lie groupoid over $M$ ); the two structures on $\Omega$ again are to commute in the sense that the maps defining the groupoid structure are all Lie algebroid morphisms (see [10]). The structures $A_{V} S$ and $A_{H} S$ associated with any

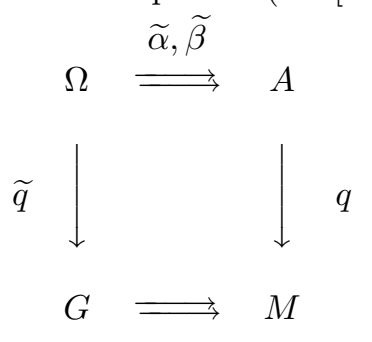

(a)

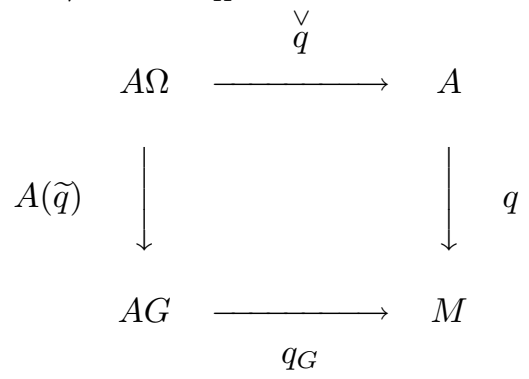

(b)

double Lie groupoid are $\mathcal{L} \mathcal{A}$-groupoids. If the Lie algebroid structures have zero bracket and zero anchor - in other words are vector bundles - then we speak of a $\mathcal{V B}$-groupoid.

Consider a Lie group $G$ equipped with an arbitrary Poisson structure $\pi^{\#}: T^{*} G \rightarrow$ $T G$. Then $T^{*} G$ has a Lie groupoid structure on base $\mathfrak{g}^{*}$ arising from the Lie group structure [5] and $T^{*} G \rightarrow G$ has a Lie algebroid structure arising from the Poisson structure. Now $(G, \pi)$ is a Poisson Lie group if and only if these two structures make $T^{*} G$ an $\mathcal{L} \mathcal{A}$-groupoid; indeed it is a Poisson Lie group if and only if $\pi^{\#}$ is a groupoid morphism [19, 4.12].

More generally, any Lie groupoid $G \Longrightarrow M$ gives rise to its cotangent groupoid $T^{*} G \Longrightarrow A^{*} G([5],[29])$ and this is a $\mathcal{V B}$-groupoid $\left(T^{*} G ; G, A^{*} G ; M\right)$. If $G$ is equipped with a Poisson structure $\pi$, then $(G, \pi)$ is a Poisson groupoid as defined by Weinstein [31] if and only if $\pi^{\#}: T^{*} G \rightarrow T G$ is a Lie groupoid morphism ([1], $[23,8.1])$, and again this is equivalent to the condition that $T^{*} G$ be an $\mathcal{L} \mathcal{A}$-groupoid with respect to the two structures.

This characterization of Poisson Lie group(oid)s is an irreducible and nonobvious fact which shows that double structures in the Ehresmann sense - the sense of this section - are fundamentally associated with Poisson group theory. We will see further confirmation of this point below.

In $[23, \S 5]$ it was shown that the structure of an arbitrary Lie algebroid $A \rightarrow M$ can be prolonged to the tangent prolongation $T A \rightarrow T M$; identifying the dual of 
$T A \rightarrow T M$ with $T\left(A^{*}\right) \rightarrow T M[23,5.3]$, the dual of this structure is the tangent lift of the Poisson structure on $A^{*}$ dual to the Lie algebroid structure of $A$.

More generally, consider an arbitrary $\mathcal{L} \mathcal{A}$-groupoid as in Figure $7(\mathrm{a})$. Applying the Lie functor as in Figure 5, we get the double vector bundle in Figure 7(b), and the vertical Lie algebroid structure in Figure 7(a) can be prolonged to the vertical bundle of (b) [20, §1].

In the case of $\Omega=T^{*} G$ for $G$ a Poisson groupoid, we obtain $A \Omega=A T^{*} G$. This is known, by a general result for symplectic groupoids ([5], or [23, 7.3]), to be isomorphic to $T^{*} A^{*} G$, the cotangent Lie algebroid of the Lie algebroid dual.

In the case of the $\mathcal{L} \mathcal{A}$-groupoids of a double Lie groupoid $S$, we have the following result.

Theorem $2.2([20,2.3])$. Let $(S ; H, V ; M)$ be a double Lie groupoid. Then $j_{S}$, regarded as a morphism of vector bundles over $A H$, is an isomorphism of Lie algebroids from the prolonged structure on $A\left(A_{V} S\right) \rightarrow A H$ to the Lie algebroid of $A_{H} S \Longrightarrow A H$.

The proof requires a substantial calculus which extends the classical complete and vertical lifting processes for vector fields. See [24] for the case $S=G \times G$, which is intermediate between the classical theory and the full proof.

Returning to the case of Poisson Lie groups, let $G^{*}$ be a Lie group integrating $\mathfrak{g}^{*}$ and suppose that the coadjoint action of $G$ on $\mathfrak{g}^{*}$ and the dressing transformation action of $\mathfrak{g}^{*}$ on $G$ integrate globally (for simple topological conditions guaranteeing this, see [16], [26]) to a left action $G \times G^{*} \rightarrow G^{*},(g, \varphi) \mapsto{ }^{g} \varphi$, and a right action $G \times G^{*} \rightarrow G,(g, \varphi) \mapsto g^{\varphi}$. Then $G \times G^{*}$ has a double Lie groupoid structure with sides $G$ and $G^{*}$ defined by regarding $(g, \varphi)$ as the empty square

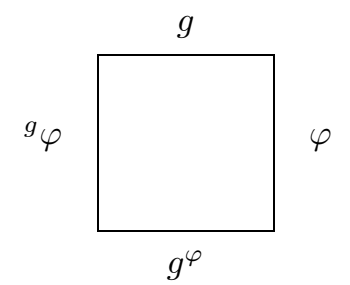

with compositions forced by Figure 3. Both groupoid structures on $S=G \times G^{*}$ are action (or transformation) groupoids and $A_{V} S=G \times \mathfrak{g}^{*} \cong T^{*} G$ as $\mathcal{L} \mathcal{A}$-groupoids. Dually, $A_{H} S \cong T^{*} G^{*}$ as $\mathcal{L} \mathcal{A}$-groupoids. See $[19, \S \S 2,4]$.

Any double groupoid $(S ; H, V ; M)$ in which a square is determined by two touching sides - a condition called vacancy in [19] — must be of this form: there are actions of $H$ on $V$ and of $V$ on $H$ which obey twisted multiplicativity conditions like those of integrated dressing transformations, and the two structures on $S$ are the corresponding action groupoids. Thus the twisted equations characteristic of dressing transformations emerge precisely from the double groupoid conditions and the simple further condition of vacancy. See [19, 2.10, 4.9].

Previous to [19], Lu and Weinstein [16] had shown that associated to any Poisson Lie group $G$ there is a (symplectic) double groupoid $\left(S ; G, G^{*} ; 1\right)$ which is locally of the form (2.1).

These results confirm in global form that the structure of double groupoids is intrinsically associated with Poisson group structures. The deep discovery by $\mathrm{Lu}$ [15] of vacant double structures associated to Poisson actions provides further evidence. 
We have so far glossed over the symplectic structure on the double groupoid of a Poisson Lie group. This has been possible since the Lie algebroid determined by the Poisson structure on the base of a symplectic groupoid coincides with the Lie algebroid of the groupoid structure [5]. However we now need to utilize these structures.

\section{Duals of Double structures}

It is well known that there is a loose equivalence between the theories of Lie algebroids and of Poisson structures: a Poisson structure on a manifold $M$ determines a Lie algebroid structure on $T^{*} M$ (see [11] for history and references), and a Lie algebroid $A$ gives rise to a (linear) Poisson structure on $A^{*}[6]$. These processes are nontrivial in the precise sense that they take place outside the original categories, involving the modules of sections of the Lie algebroids and the cotangents and tangents (or rings of functions) of the Poisson manifolds. It is consequently often valuable to convert problems of Lie algebroid theory into Poisson terms, and vice versa.

The symplectic groupoid $T^{*} G \Longrightarrow A^{*} G$, where $G \Longrightarrow M$ is any Lie groupoid, is fundamental to integrability problems in Poisson geometry. Constructed first by Coste, Dazord and Weinstein [5], it was obtained by Pradines [29] as an instance of a general duality process applied to the much simpler tangent prolongation groupoid $T G \Longrightarrow T M$. This duality, as we shall see, provides a systematic means of handling cotangent structures, despite their nonfunctoriality.

The cotangent groupoid provides the simplest important example of symplectic groupoids, but it is also necessary for the definition of Poisson groupoid which is most useful here: a Lie groupoid $G$ with a Poisson structure $\pi^{\#}: T^{*} G \rightarrow T G$ is a Poisson groupoid if $\pi^{\#}$ is a groupoid morphism ([1], [23, 8.1]).

We will see that it is correspondingly necessary, for the understanding of a double groupoid $(S ; H, V ; M)$, to place a double groupoid structure on $T^{*} S$. Since $S$ has two groupoid structures, $T^{*} S$ has two cotangent groupoid structures, over $A_{H}^{*} S$ and $A_{V}^{*} S$. We must show that these duals are themselves groupoids over a common base.

Consider a $\mathcal{V B}$-groupoid $(\Omega ; G, A ; M)$ as in Figure $7(\mathrm{a})$, and let $K$ denote the intersection of the kernels of the source projection $\Omega \rightarrow A$ and the bundle projection $\Omega \rightarrow G$. Call $K$ the core of $\Omega$; it has a vector bundle structure over $M$ obtained from that in $\Omega \rightarrow G[19, \S 4]$. Pradines [29] defined a groupoid structure on the vector bundle $\Omega^{*}$ with base $K^{*}$ which makes $\left(\Omega^{*} ; G, K^{*} ; M\right)$ a $\mathcal{V B}$-groupoid with core $A^{*}$. If moreover $\Omega$ is an $\mathcal{L} \mathcal{A}$-groupoid, then the core has a natural induced Lie algebroid structure $[19, \S 4]$. When $\Omega=T G$ the core is $A G$ and the dual $\mathcal{V B}$-groupoid defines the cotangent groupoid structure.

For a double Lie groupoid $S$, Brown and the author [4] defined the core $C$ to be the intersection of the kernels of the source projections. There is a canonical groupoid structure on $C$ with base $M$ which is induced by (but is not a restriction of) the structures on $S$. In the presence of a double version of local triviality, $C$ and its associated structure actually determine $S$.

Finally, the $\mathcal{L} \mathcal{A}$-groupoids $A_{V} S$ and $A_{H} S$ both have core $A C$, and both induce the usual Lie algebroid structure on $A C([20,1.6])$. It therefore follows that the dual $\mathcal{L} \mathcal{A}$-groupoids are $\left(A_{V}^{*} S ; H, A^{*} C ; M\right)$ and $\left(A_{H}^{*} S ; A^{*} C, V ; M\right)$. The first part of the following theorem is a long but routine check. 
Theorem $3.1([21,1.4,1.5])$. With the structures above, $\left(T^{*} S ; A_{V}^{*} S ; A_{H}^{*} S ; A^{*} C\right)$ is a double Lie groupoid, and has core groupoid $T^{*} C \Longrightarrow A^{*} C$.

With its canonical symplectic structure, $T^{*} S$ is the model of a symplectic double groupoid. More generally, we define a Poisson double groupoid to be a double Lie groupoid $(\mathcal{S} ; \mathcal{H}, \mathcal{V} ; P)$ with a Poisson structure $\pi^{\#}: T^{*} \mathcal{S} \rightarrow T \mathcal{S}$ which is a morphism of double groupoids from $T^{*} \mathcal{S}$ to the tangent double groupoid $(T \mathcal{S} ; T \mathcal{H}, T \mathcal{V} ; T P)$ [21]. It then follows that $\mathcal{H}$ and $\mathcal{V}$ are Poisson groupoids, with respect to the induced structures, both inducing the same Poisson structure on $P$. Further, taking into account the bundle structures of $T^{*} \mathcal{S}$ and $T \mathcal{S}$ and treating them as triple structures, the restriction of $\pi^{\#}$ to certain corners and cores gives Lie algebroid morphisms $D_{V}: A^{*} \mathcal{H} \rightarrow A \mathcal{V}$ and $D_{H}: A^{*} \mathcal{V} \rightarrow A \mathcal{H}$ with $D_{H}^{*}=-D_{V}$. When $\pi^{\#}$ is symplectic these are both isomorphisms of Lie algebroids, and $\mathcal{H}$ and $\mathcal{V}$ are Poisson groupoids in duality in the sense of Weinstein $[31, \S 4.5]$. Taking $\mathcal{S}=T^{*} S$ gives the following crucial result.

Theorem 3.2 ([21, 2.12]). Let $(S ; H, V ; M)$ be a double Lie groupoid with core $C$. Then $A_{V}^{*} S \Longrightarrow A^{*} C$ and $A_{H}^{*} S \Longrightarrow A^{*} C$ are Poisson groupoids in duality.

The duality isomorphisms $A^{*}\left(A_{H}^{*} S\right) \rightarrow A\left(A_{V}^{*} S\right)$ and $A^{*}\left(A_{V}^{*} S\right) \rightarrow A\left(A_{H}^{*} S\right)$ can be described in terms which generalize the description in $[23,7.3]$ of the canonical symplectic structure on a cotangent bundle in terms of the Tulczyjew isomorphism $\alpha: T^{*} T M \rightarrow T T^{*} M$ and the map $R$ mentioned in $\S 1$. See [21,3.9].

We have therefore associated to any double Lie groupoid a pair of dual Lie bialgebroids with base $A^{*} C$. This is the key to the results of $\S 4$.

\section{Lie Bialgebroids AND Double Lie ALGebroids}

We suppose given a double vector bundle [28] as in Figure 8; that is, each side has a vector bundle structure, and the two structures on $\mathcal{A}$ commute in the sense that

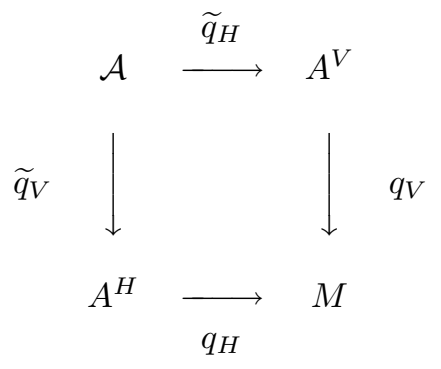

FiguRE 8.

the maps defining each structure on $\mathcal{A}$ (the bundle projection, zero section, addition and scalar multiplication) are morphisms with respect to the other. We suppose given Lie algebroid structures on each of the four sides, and want to describe what it means for the two Lie algebroid structures on $\mathcal{A}$ to commute. There are certain obvious requirements, which need no explanation. To express these efficiently, define an $\mathcal{L} A$-vector bundle to be a double vector bundle as in Figure 8 together with Lie algebroid structures on a pair of parallel sides, such that the structure maps of the other pair of vector bundle structures are Lie algebroid morphisms.

The definition of double Lie algebroid is made up of three conditions. 
Condition I. With respect to the two vertical Lie algebroids, $\mathcal{A} \rightarrow A^{H}$ and $A^{V} \rightarrow$ $M$, the double vector bundle $\mathcal{A}$ is an $\mathcal{L} A$-vector bundle. Likewise, with respect to the two horizontal Lie algebroids, $\mathcal{A} \rightarrow A^{V}$ and $A^{H} \rightarrow M$, the double vector bundle $\mathcal{A}$ is an $\mathcal{L} A$-vector bundle.

Denote the four anchors by $\widetilde{a}_{V}: \mathcal{A} \rightarrow T\left(A^{H}\right), \widetilde{a}_{H}: \mathcal{A} \rightarrow T\left(A^{V}\right), a_{V}: A^{V} \rightarrow T M$ and $a_{H}: A^{H} \rightarrow T M$. It is automatic that $\widetilde{a}_{V}$ is a morphism of Lie algebroids over the fixed base $A^{H}$, and that $a_{V}$ is a morphism of Lie algebroids over $M$.

Condition II. The anchors $\widetilde{a}_{V}$ and $a_{V}$ form a morphism $\left(\widetilde{a}_{V}, a_{V}\right)$ of Lie algebroids with respect to the horizontal structure on $\mathcal{A}$ and the prolongation to $T A^{H} \rightarrow$ $T M$ of the structure on $A^{H} \rightarrow M$. Likewise, the anchors $\widetilde{a}_{H}$ and $a_{H}$ form a morphism of Lie algebroids with respect to the vertical structure on $\mathcal{A}$ and the prolongation to $T A^{V} \rightarrow T M$ of the structure on $A^{V} \rightarrow M$.

We come now to the main condition. The duality for $\mathcal{V B}$-groupoids of $\S 3$ can be applied to either structure on $\mathcal{A}$ and yields a vertical dual $\mathcal{A}^{* V}$ and a horizontal dual $\mathcal{A}^{* H}$, which are themselves double vector bundles as in Figure 9 . Here $K$ is the core of $\mathcal{A}$, and the cores of $\mathcal{A}^{* V}$ and $\mathcal{A}^{* H}$ are $\left(A^{V}\right)^{*}$ and $\left(A^{H}\right)^{*}$ respectively. Remarkably, $\mathcal{A}^{* V}$ and $\mathcal{A}^{* H}$ are themselves dual.

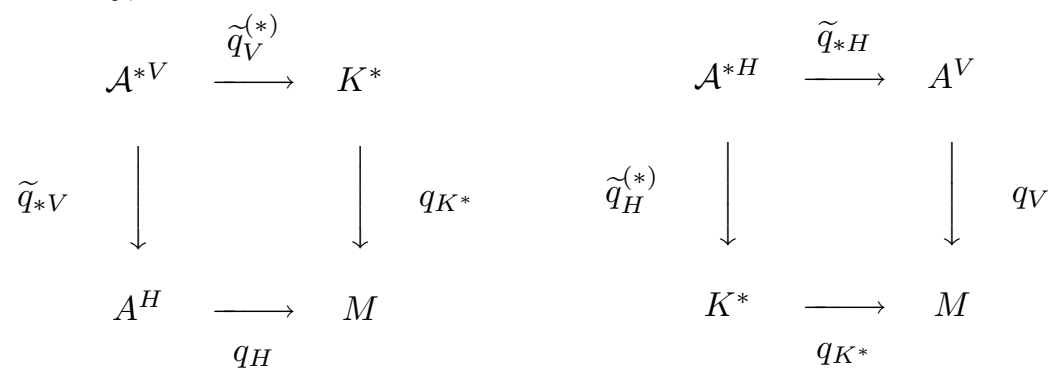

(a)

FiguRE 9.

(b)

Theorem 4.1 ([21, 3.1, 3.5]). There is a nondegenerate pairing of the bundles $\mathcal{A}^{* V} \rightarrow K^{*}$ and $\mathcal{A}^{* H} \rightarrow K^{*}$ given by

$$
\langle\Phi, \Psi\rangle=\langle\Psi, \xi\rangle-\langle\Phi, \xi\rangle
$$

where $\Phi \in \mathcal{A}^{* V}, \Psi \in \mathcal{A}^{* H}$ have $\widetilde{q}_{V}^{(*)}(\Phi)=\widetilde{q}_{H}^{(*)}(\Psi)$ and $\xi$ is any element of $\mathcal{A}$ with $\widetilde{q}_{V}(\xi)=\widetilde{q}_{* V}(\Phi)$ and $\widetilde{q}_{H}(\xi)=\widetilde{q}_{* H}(\Psi)$.

The induced isomorphism $Z_{V}:\left(\mathcal{A}^{* H}\right)^{\dagger} \rightarrow \mathcal{A}^{* V}$, where ${ }^{\dagger}$ denotes the dual over $K^{*}$, is an isomorphism of double vector bundles over $A^{H}$ and $K^{*}$, inducing-id on the cores $\left(A^{V}\right)^{*}$.

This theorem has also been found very recently by Konieczna and Urbański [12]. Note that the pairing on the LHS of (4.1) is over $K^{*}$, whereas the pairings on the RHS are over $A^{V}$ and $A^{H}$ respectively. When $\mathcal{A}=T A$ as in Figure 2(b), $Z_{V}=R \circ I^{\dagger}$ in terms of the maps $R$ and $I$ of $[23, \S 5]$.

It follows from Condition I and $[21,3.14]$ that the Poisson structure on $\mathcal{A}^{* H}$, which is automatically linear over $A^{V}$, is also linear over $K^{*}$, and therefore induces a Lie algebroid structure on its dual $\left(\mathcal{A}^{* H}\right)^{\dagger}$. We use $Z_{V}$ to transfer this to $\mathcal{A}^{* V} \rightarrow$ $K^{*}$. Similarly the Poisson structure on $\mathcal{A}^{* V}$ is linear over $K^{*}$ and therefore induces a Lie algebroid structure on $\left(\mathcal{A}^{* V}\right)^{\dagger} \rightarrow K^{*}$ which may be transferred to $\mathcal{A}^{* H}$. 
Condition III. With respect to these structures, $\left(\mathcal{A}^{* V}, \mathcal{A}^{* H}\right)$ is a Lie bialgebroid over $K^{*}$. Further, $\left(\mathcal{A}^{* V} ; A H, K^{*} ; M\right)$ is an $\mathcal{L} A$-vector bundle with respect to the horizontal Lie algebroid structures and $\left(\mathcal{A}^{* H} ; K^{*}, A V ; M\right)$ is an $\mathcal{L} A$-vector bundle with respect to the vertical structures.

Definition $4.2([22])$. A double Lie algebroid is a double vector bundle as in Figure 8 equipped with Lie algebroid structures on all four sides such that the above Conditions I, II, III are satisfied.

When $\left(\mathcal{A} ; A^{H}, A^{V} ; M\right)$ is a double Lie algebroid we call $\left(\mathcal{A}^{* V}, \mathcal{A}^{* H}\right)$ the associated Lie bialgebroid.

The notion of Lie bialgebroid was defined by the author and $\mathrm{Xu}$ in [23] in terms of the coboundary operators associated to $A$ and to $A^{*}$; a more efficient and elegant reformulation was then given by Kosmann-Schwarzbach in [13]. The criterion most useful in the current setting is the following.

Theorem 4.3 ([23, 6.2]). Let $A$ be a Lie algebroid on $M$ such that its dual vector bundle $A^{*}$ also has a Lie algebroid structure. Denote their anchors by $a, a_{*}$. Then $\left(A, A^{*}\right)$ is a Lie bialgebroid if and only if

$$
T^{*}\left(A^{*}\right) \stackrel{R}{\longrightarrow} T^{*}(A) \stackrel{\pi_{A}^{\#}}{\longrightarrow} T A
$$

is a Lie algebroid morphism over $a_{*}$, where the domain $T^{*}\left(A^{*}\right) \rightarrow A^{*}$ is the cotangent Lie algebroid induced by the Poisson structure on $A^{*}$, and the target $T A \rightarrow T M$ is the tangent prolongation of $A$.

Example $4.4([22])$. Let $(S ; H, V ; M)$ be a double Lie groupoid. By $3.2, A_{H}^{*} S$ and $A_{V}^{*} S$, the duals of the $\mathcal{L} \mathcal{A}$-groupoids of $S$, are a dual pair of Poisson groupoids over $A^{*} C$ with respect to the dual Poisson structures. Further [21, $\left.\S 3\right], A^{*}\left(A_{H} S\right)$, the vertical dual of $A_{2} S$, is canonically isomorphic to $A\left(A_{V}^{*} S\right)$; this canonical isomorphism extends the well-known $T^{*} T M \cong T T^{*} M$ of [30]. Identifying $A_{2} S$ with $A^{2} S$, the horizontal dual is $A^{*}\left(A_{V} S\right) \cong A^{*}\left(A_{V}^{*} S\right)$, by a generalization of the map $R$. Thus $\left(\mathcal{A}^{* V}, \mathcal{A}^{* H}\right)$ can be identified with the Lie bialgebroid of $A_{V}^{*} S \Longrightarrow A^{*} C$, which establishes Condition III.

More generally, dualizing any $\mathcal{L} \mathcal{A}$-groupoid $(\Omega ; G, A ; M)$ gives a Poisson groupoid $\Omega^{*} \Longrightarrow K^{*}[21,3.14]$ whose Lie bialgebroid shows that $(A \Omega ; A G, A ; M)$, as in Figure $7(\mathrm{~b})$, is a double Lie algebroid.

We come now to the concept of double for a Lie bialgebroid. It was shown in $[21,2.11]$ that if $G \Longrightarrow P$ is a Poisson groupoid arising from a symplectic double groupoid $\left(S ; G, G^{*} ; P\right)$, then the double Lie algebroid of $S$ is the double cotangent $T^{*}(A G) \cong T^{*}\left(A G^{*}\right) \cong T^{*}\left(A^{*} G\right)$. For a Poisson group $G$, such a symplectic double groupoid always exists [16]. In the Poisson groupoid case $G^{*}$ may not exist (but see [25]) and it seems unlikely that, even when it does, a symplectic double groupoid $S$ for $G$ and $G^{*}$ necessarily exists. We therefore wish to formulate the double cotangent structure without assuming that the Lie bialgebroid comes from a Poisson groupoid.

Let $A$ be a Lie algebroid on $M$ such that $A^{*}$ has a Lie algebroid structure, not a priori related to that on $A$. The structure on $A^{*}$ induces a Poisson structure on $A$, and this gives rise to a cotangent Lie algebroid $T^{*} A \rightarrow A$. Equally, the Lie algebroid structure on $A$ induces a Poisson structure on $A^{*}$ and this gives rise to a cotangent Lie algebroid $T^{*} A^{*} \rightarrow A^{*}$. We transfer this latter structure to $T^{*} A \rightarrow A^{*}$ via $R$. 
There are now four Lie algebroid structures on the four sides of $\mathcal{A}=T^{*} A$ as in Figure 1.

Theorem 4.5 ([22]). Let $A$ be a Lie algebroid on $M$ such that its dual vector bundle $A^{*}$ also has a Lie algebroid structure. Then $\left(A, A^{*}\right)$ is a Lie bialgebroid if and only if $\mathcal{A}=T^{*} A$, with the structures just described, is a double Lie algebroid.

When $\left(A, A^{*}\right)$ is a Lie bialgebroid, the Lie bialgebroid associated to its double is effectively $\left(T A, T\left(A^{*}\right)\right)$, the tangent prolongation over $T M$. The Manin triple theorem as given by $[17, \S 1]$ characterizes a Lie bialgebra in terms of the structure of the double on $\mathfrak{g} \oplus \mathfrak{g}^{*}$ built out of the given structures on $\mathfrak{g}$ and $\mathfrak{g}^{*}$ and the two coadjoint representations. In the algebroid case the presence of the core $T^{*} M$ precludes a corresponding structure over the base $M$, and we have used instead the side structures over $A$ and $A^{*}$. (It is shown in $[19, \S 2]$ that the diagonal structure associated with a matched pair of groupoids, defined in terms of analogues of the dressing transformation actions, is a consequence of the triviality of the core; the cotangent double in Figure 1 has trivial core if and only if the base is trivial.) By considering the duality (4.1) one can show that a double Lie algebroid as in Figure 8, for which both structures on $\mathcal{A}$ are cotangents, must be of the form of Figure 1.

The results treated here show that double Lie algebroids stand in the same relationship to Lie bialgebroids as ordinary Lie algebroids do to general Poisson manifolds. Any (ordinary) Lie algebroid gives rise to a Poisson structure on its dual - a Poisson structure which is necessarily linear - and any Poisson manifold gives rise to a Lie algebroid structure on its cotangent. These processes are not equivalences of categories, but it is nonetheless very valuable to be able to pass from one setting to the other by means of them. It is in the same sense that we say that Drinfel'd doubles and Ehresmann doubles are coextensive: Theorem 4.5 shows that the Drinfel'd double of a Lie bialgebroid has a double structure in the sense of Ehresmann, and conversely 3.2 and Condition III of 4.2 demonstrate that every double Lie groupoid or double Lie algebroid - where "double" is now used in the Ehresmann sense - has hidden in its structure as a key ingredient, a dual pair of Lie bialgebroids and their Drinfel'd double.

We have concentrated here on the characterization of Lie bialgebroids in terms of their double cotangent Lie algebroids. However, there are a number of other characterization results for particular classes of double Lie algebroids that could be given at this stage. Firstly, it can be shown [22] that if a double Lie algebroid is vacant - that is, the core is the zero bundle - then it defines a matched pair structure (as in Mokri [27]) on the two side Lie algebroids. Whereas matched pairs of groups and groupoids can be defined in terms of conditions on their elements, this characterization is in terms of diagrams of maps, and may well be capable of extension to other contexts arising in quantization.

It is also clear that some of the unexpected features of the work of $\mathrm{Lu}$ [15] on Poisson homogeneous spaces can be understood in terms of the dualities studied here.

Lastly, we believe that the calculus developed by Liu, Weinstein and Xu [14] will emerge naturally from the study of the double and triple structures introduced here. This is the subject of ongoing work. 


\section{REFERENCES}

[1] C. Albert and P. Dazord. Théorie des groupoïdes symplectiques: Chapitre II, Groupoïdes symplectiques. In Publications du Département de Mathématiques de l'Université Claude Bernard, Lyon I, nouvelle série, pages 27-99, 1990. MR 95m:58134

[2] A. L. Besse. Manifolds all of whose geodesics are closed, volume 93 of Ergebnisse der Mathematik und ihrer Grenzgebiete. Springer-Verlag, 1978. MR 80c:53044

[3] R. Brown. From groups to groupoids: a brief survey. Bull. London Math. Soc., 19:113-134, 1987. MR 87m:18009

[4] R. Brown and K. C. H. Mackenzie. Determination of a double Lie groupoid by its core diagram. J. Pure Appl. Algebra, 80(3):237-272, 1992. MR 93g:55022

[5] A. Coste, P. Dazord, and A. Weinstein. Groupoïdes symplectiques. In Publications du Département de Mathématiques de l'Université de Lyon, I, number 2/A-1987, pages 1-65, 1987. MR 90g:58033

[6] T. J. Courant. Dirac manifolds. Trans. Amer. Math. Soc., 319:631-661, 1990. MR 90m:58065

[7] V. G. Drinfel'd. Hamiltonian structures on Lie groups, Lie bialgebras and the geometric meaning of the classical Yang-Baxter equation. Soviet. Math. Dokl., 27:68-71, 1983. MR 84i: 58044

[8] V. G. Drinfel'd. Quantum groups. In A. M. Gleason, editor, Proceedings of the International Congress of Mathematicians, Berkeley, 1986, pages 798-820. American Mathematical Society, Providence, RI, 1987. MR 89f: 17017

[9] A. C. Ehresmann, editor. Charles Ehresmann: Euvres complètes et commentées. Seven volumes. Imprimerie Evrard, Amiens, 1984.

[10] P. J. Higgins and K. C. H. Mackenzie. Algebraic constructions in the category of Lie algebroids. J. Algebra, 129:194-230, 1990. MR 92e:58241

[11] J. Huebschmann. Poisson cohomology and quantization. J. Reine Angew. Math., 408:57-113, 1990. MR 92e: 17027

[12] K. Konieczna and P. Urbański. Double vector bundles and duality. Preprint. dg-ga/9710014.

[13] Y. Kosmann-Schwarzbach. Exact Gerstenhaber algebras and Lie bialgebroids. Acta Appl. Math., 41:153-165, 1995. MR 97i:17021

[14] Zhang-Ju Liu, Alan Weinstein, and Ping Xu. Manin triples for Lie bialgebroids. J. Differential Geom., 45:547-574, 1997. MR 98f:58203

[15] Jiang-Hua Lu. Poisson homogeneous spaces and Lie algebroids associated to Poisson actions. Duke Math. J., 86:261-304, 1997. MR 98d:58204

[16] Jiang-Hua Lu and A. Weinstein. Groupoïdes symplectiques doubles des groupes de LiePoisson. C. R. Acad. Sci. Paris Sér. I Math., 309:951-954, 1989. MR 91i:58045

[17] Jiang-Hua Lu and A. Weinstein. Poisson Lie groups, dressing transformations, and Bruhat decompositions. J. Differential Geom., 31:501-526, 1990. MR 91c:22012

[18] K. Mackenzie. Lie groupoids and Lie algebroids in differential geometry. London Mathematical Society Lecture Note Series, no. 124. Cambridge University Press, 1987. MR 89g:58225

[19] K. C. H. Mackenzie. Double Lie algebroids and second-order geometry, I. Adv. Math., 94(2):180-239, 1992. MR 93f:58255

[20] K. C. H. Mackenzie. Double Lie algebroids and iterated tangent bundles. Submitted, 1998. $27 \mathrm{pp}$.

[21] K. C. H. Mackenzie. On symplectic double groupoids and duality for Poisson groupoids. Submitted, 1998. 21pp.

[22] K. C. H. Mackenzie. Double Lie algebroids and the double of a Lie bialgebroid. Preprint, 1998. 25pp.

[23] K. C. H. Mackenzie and Ping Xu. Lie bialgebroids and Poisson groupoids. Duke Math. J., 73(2):415-452, 1994. MR 95b:58171

[24] K. C. H. Mackenzie and Ping Xu. Classical lifting processes and multiplicative vector fields. Quarterly J. Math. Oxford (2), 49:59-85, 1998. CMP 98:11

[25] K. C. H. Mackenzie and Ping Xu. Integrability of Lie bialgebroids. Submitted, 1997.

[26] S. Majid. Matched pairs of Lie groups associated to solutions of the Yang-Baxter equations. Pacific J. Math., 141:311-332, 1990. MR 91a:17009

[27] T. Mokri. Matched pairs of Lie algebroids. Glasgow Math. J., 39:167-181, 1997. CMP 97:15

[28] J. Pradines. Fibrés vectoriels doubles et calcul des jets non holonomes. Notes polycopiées, Amiens, 1974. MR 83b:58010 
[29] J. Pradines. Remarque sur le groupoïde cotangent de Weinstein-Dazord. C. R. Acad. Sci. Paris Sér. I Math., 306:557-560, 1988. MR 89h:58222

[30] W. M. Tulczyjew. Geometric formulation of physical theories, volume 11 of Monographs and Textbooks in Physical Science. Bibliopolis, Naples, 1989. MR 91d:58084

[31] A. Weinstein. Coisotropic calculus and Poisson groupoids. J. Math. Soc. Japan, 40:705-727, 1988. MR 90b:58091

School of Mathematics and Statistics, University of Sheffield, Sheffield, S3 7RH, ENGLAND

E-mail address: K.Mackenzie@sheffield.ac.uk 